\title{
GaN micro-light-emitting diode arrays with monolithically integrated sapphire microlenses
}

\author{
H. W. Choi, ${ }^{\text {a) }}$ C. Liu, and E. Gu \\ Institute of Photonics, University of Strathclyde, Wolfson Centre, 106 Rottenrow, Glasgow G4 ONW, \\ United Kingdom \\ G. McConnell \\ Centre for Biophotonics, University of Strathclyde, 27 Taylor Street, Glasgow G4 ONR, United Kingdom \\ J. M. Girkin, I. M. Watson, and M. D. Dawson \\ Institute of Photonics, University of Strathclyde, Wolfson Centre, 106 Rottenrow, Glasgow G4 ONW, \\ United Kingdom
}

(Received 24 October 2003; accepted 3 February 2004)

\begin{abstract}
GaN micro-light-emitting diodes (micro-LEDs) with monolithically integrated microlenses have been demonstrated. Microlenses, with a focal length of $44 \mu \mathrm{m}$ and a root mean square roughness of $\sim 1 \mathrm{~nm}$, have been fabricated on the polished back surface of a sapphire substrate of an array of micro-LEDs by resist thermal reflow and plasma etching. The optical properties of the microlenses have been demonstrated to alter the emission pattern of the LED emitters. The cone of light emitted from this hybrid device is significantly less divergent than a conventional broad-area device. This combination of micro-LED and microlens technologies offers the potential for further improvement in the overall efficiency of GaN-based light emitters. (C) 2004 American Institute of Physics.
\end{abstract}

[DOI: 10.1063/1.1690876]

A wide range of approaches in epitaxy, processing and packaging has been explored in order to improve the efficiency of gallium-nitride-based light-emitting diodes. Advanced epitaxial techniques have produced multiquantum well (MQW) structures with external quantum efficiency as high as $30 \% .^{1}$ In terms of device processing, one breakthrough has been the practical introduction of the concept of arrays of micro-light-emitting diodes (micro-LEDs). ${ }^{2,3}$ Such micro-LEDs, consisting of arrays of emitter elements with typical individual diameters of 4-20 $\mu \mathrm{m}$, have proven effective in increasing the extraction efficiency through enhanced sidewall area and reduced optical (re)absorption. ${ }^{4}$ Recently, flip-chip packages have been adopted for gallium-nitride LEDs, ${ }^{5}$ enabling the extraction of light through the transparent sapphire substrate, as opposed to light extraction through a semitransparent current spreading layer on the device surface. Flip-chip packages for the nitride devices are complicated and costly, however, this approach offers potential use of a sapphire substrate, which is commonly used for nitride metalorganic chemical vapor deposition (MOCVD) growth, as a material in which optical elements can be integrated into optical devices.

In this letter, the concept of micro-LEDs and light extraction through the sapphire face are combined into a single device. Furthermore, microlenses have been integrated into the device in registry with individual micro-LED emitting elements in order to improve the directionality and to demonstrate optical control of the light emitted.

The micro-LED arrays were fabricated on MOCVDgrown InGaN/GaN LED wafers using sapphire as the substrate and multiquantum wells designed for light emission at

a) Author to whom correspondence should be addressed; electronic mail: anthony.choi@strath.ac.uk
$470 \mathrm{~nm}$. The back side of the sapphire was polished for optimal light extraction. Details of the micro-LED fabrication process are reported elsewhere. ${ }^{3,6}$ Microlenses were fabricated on the polished sapphire surface at locations aligned to emitters that formed the micro-LED array. The microlenses were fabricated using a thermal resist reflow technique. ${ }^{7}$ Photoresist microdisks with a nominal thickness of $7 \mu \mathrm{m}$ were patterned by standard photolithography and subsequently shaped into a lenticular shape on a hotplate at $115^{\circ} \mathrm{C}$ for $15 \mathrm{~min}$, and the lens pattern was transferred into the sapphire by inductively coupled plasma (ICP) dry etching. A maximum etch rate, based on a $\mathrm{Cl}_{2} / \mathrm{BCl}_{3} / \mathrm{Ar}$ plasma, of $120 \mathrm{~nm} / \mathrm{min}$ was achieved.

The $I-V$ characteristics of the devices were measured with a HP4145 parametric analyzer while the profile and smoothness of the microlenses were examined by atomic force microscopy (AFM) in the tapping mode utilizing a Digital Instruments multimode Nanoscope IIIa. The resulting radiation pattern of light emission from the combined LED and lens array was recorded using a rotating a light detector centered on the axis of the device. In a specially designed jig the LED array was held at the center of a circle about which a photodiode detector was constrained to move. The silicon detector was mounted behind a $1 \mathrm{~mm}$ aperture to give an angular resolution of $5^{\circ}$ for the distribution of light.

In order to determine the optical properties of individual elements of the microlens array an array of microlenses was fabricated, using the same technique, on a separate doublesided polished $c$-plane sapphire substrate. The diameter and height of the fabricated microlenses were 20 and $1.5 \mu \mathrm{m}$, respectively, measured using AFM, giving a calculated focal length of $43 \mu \mathrm{m}$ using the method described by Oder et al. ${ }^{8}$ based on a refractive index of 1.8 for sapphire at a wavelength of $470 \mathrm{~nm}^{9}$ An AFM image of a microlens array is 


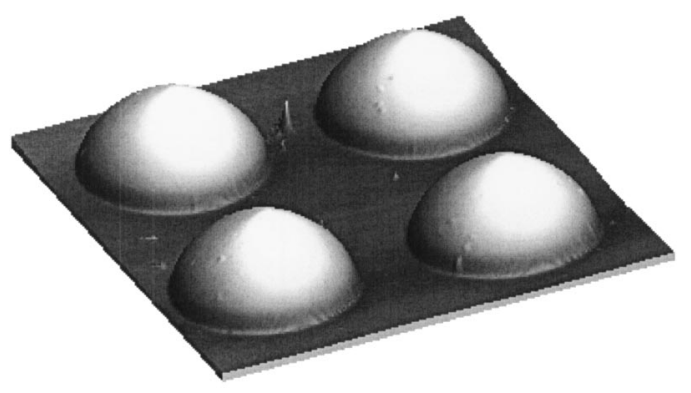

FIG. 1. $50 \times 50 \mu \mathrm{m}^{2}$ AFM plot of a microlens array plasmaetched onto sapphire. The rms roughness was determined to be $1 \mathrm{~nm}$.

shown in Fig. 1. From these plots the root mean square (rms) roughness of the lenses was determined to be better than 1 $\mathrm{nm}$, or $\lambda / 470$, ensuring high optical quality.

The optical performance of the lenses was evaluated using a technique that utilized a confocal microscope operating in both reflection and transmission mode through the lens array. Full details of this technique will be published elsewhere. A cross-sectional view of the combined confocal image is shown in Fig. 2, demonstrating both the reflection from the lens surface and the focusing power of the lens on the light transmitted. The images were analyzed using commercial software (Metamorph), giving an effective focal distance of $44( \pm 1.5 \mu \mathrm{m})$ from the apex of the convex surface of the lens array. This is in good agreement with the value calculated from the curvature measured using the AFM data.

For the integrated monolithic device, a microlens array was fabricated on the back side of a microdisk LED, as illustrated in the detailed schematic in Figs. 3(a) and 3(b). Note that thick Ni/Au metal bilayers $(20 \mathrm{~nm} / 300 \mathrm{~nm})$ were employed as the current spreading layer for contact, and it doubles as a light reflector. The locations of the microlenses matched those of the micro-LED emitters. However, due to the difficulties of aligning two different planes $350 \mu \mathrm{m}$ apart on either side of the sapphire substrate, an error of $\pm 2 \mu \mathrm{m}$ can be expected. As a result, light emitted from the MQW, together with the light reflected from the bottom metal mirror, could be collected by the sapphire microlenses. By extracting blue light from the sapphire side $(n=1.8$ at $470 \mathrm{~nm})$ instead of the GaN side ( $n=2.4$ at $470 \mathrm{~nm})$, an increase in extraction efficiency of approximately $15 \%$ can be expected.

Nevertheless, the integration of microlenses into the device does not significantly affect its extraction efficiency. The $L-I$ characteristics of the LED with a lens, compared to a conventional broad-area LED without a lens with an identical light-generation area, ${ }^{3}$ are shown in Fig. 4. Light was collected with a Si detector situated $5 \mathrm{~mm}$ above the emission surface of the devices. Although more light was col-

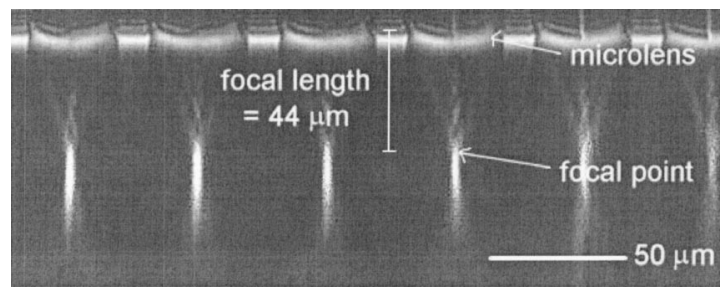

FIG. 2. Cross-sectional image showing light pathways through the sapphire

microlens array obtained by confocal microscopy.
Downloaded 09 Nov 2006 to 147.8 .21 .97 . Redistribution subject to AlP license or copyright, see http://ap crolenses and those of a broad-area LED.

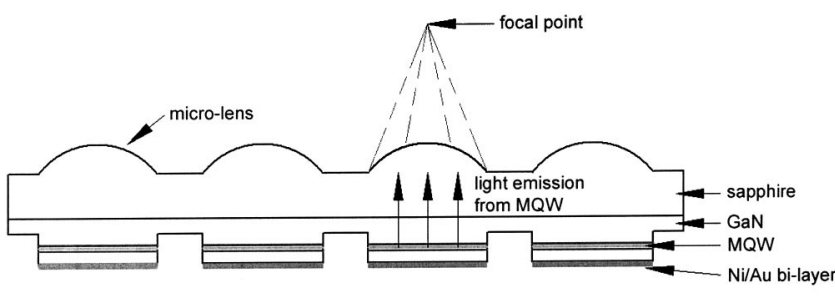

(a)

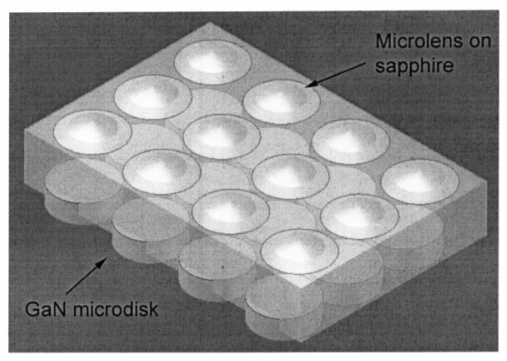

(b)

FIG. 3. (a) Schematic diagram of the device, showing the microlens array on the sapphire face and the microdisk array on the GaN face. (b) Threedimensional image of the hybrid structure.

lected from the LED with microlenses, this is mainly attributed to light extraction from the sapphire surface. In fact, $23 \%$ more light was collected from the LED with a lens at $I=20 \mathrm{~mA}$, higher than the theoretical prediction of $15 \%$. This is mainly due to the directionality of the light emitted.

The main advantage of this integrated device lies in its emission characteristics. A plot of the angular distribution of the normalized intensity emission from the device is shown in Fig. 5, and is compared to the light emission characteristics of a conventional broad-area device with light emission from the top surface. The peak output of each device was normalized to illustrate the relative spread of the two outputs. It can be seen that the angle of emission from the device is significantly reduced compared to the device without a lens. The energy output from the broad-area device is uniformly distributed, with a full emission angle of nearly $180^{\circ}$, and an intensity of $80 \%$ of the peak even at an emission

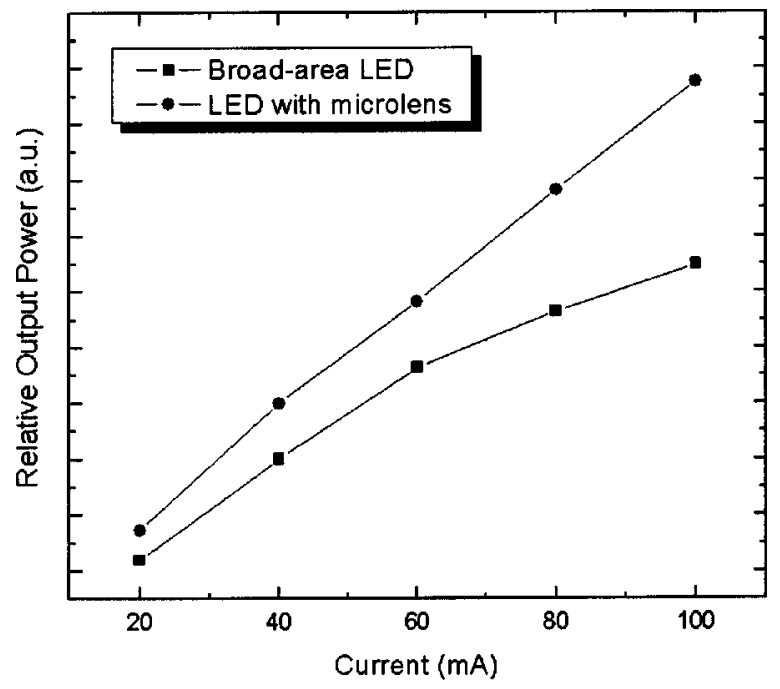

FIG. 4. Comparison of $L-I$ characteristics of the microdisk LED with mi- 


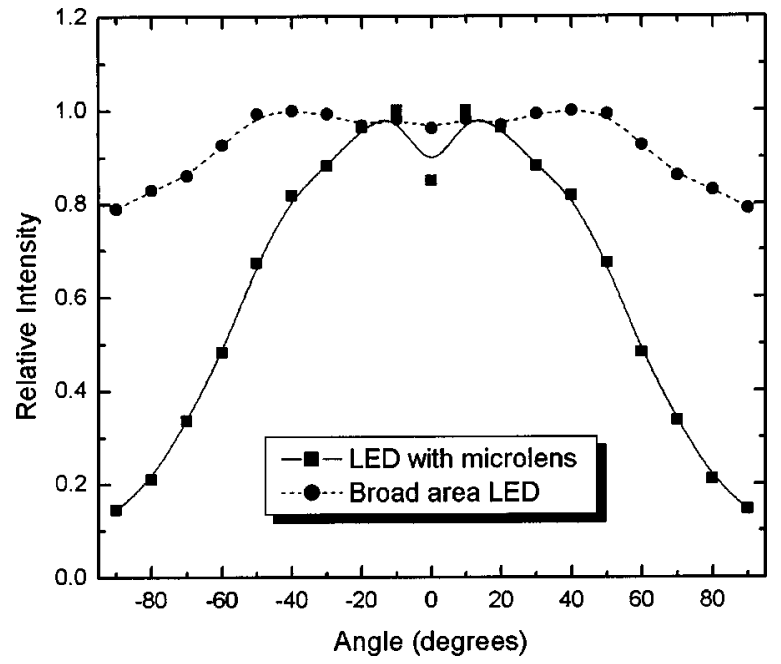

FIG. 5. Comparison of the angular distribution of light emitted from a microlens-microdisk LED and from a broad-area device.

angle of $80^{\circ}$. In contrast, the microlens array collects light emitted from the MQWs in the microdisks and concentrates the emission into a direction around the normal to the plane of the quantum well, resulting in less than $20 \%$ of the light being emitted at angles greater than $40^{\circ}$. The emission is thus more intense and can subsequently be manipulated with optics if required. In order to make light from the broad-area device more suitable for practical applications bulk macrooptics are frequently required in the LED mount. Having the optical elements integrated into the device ensures minimal loss along optical pathways. As a result, the need for external lenses may be eliminated and the package can thus be simplified, with the potential benefit being a significant reduction in the cost of the packaging process.

Further optimization in the design of the device is cur- rently under investigation in order to further improve the directionality of light emission. Some considerations include the optical pathway between the light emission region and the microlenses (by reducing the thickness of the sapphire substrate by lapping) and tailoring of the focal length of the microlenses for specific purposes.

In summary, a microdisk LED with an integrated microlens array was demonstrated. Despite the hardness of the sapphire substrate, a microlens array was transferred onto the back side of the polished LED sample, with rms roughness of better than $1 \mathrm{~nm}$. The focal lengths of the microlenses were determined experimentally to be around $44 \mu \mathrm{m}$. The capability of the lenses in concentrating light emitted from microdisk LEDs was demonstrated. Such monolithic integration of micro-optic elements eliminates the need for external optics and thus increases the overall efficiency of the optical system.

${ }^{1}$ M. Koike, N. Shibata, H. Kato, and Y. Takahashi, IEEE J. Sel. Top. Quantum Electron. 8, 271 (2002).

${ }^{2}$ S. X. Jin, J. Li, J. Z. Li, J. Y. Lin, and H. X. Jiang, Appl. Phys. Lett. 76, 631 (2000).

${ }^{3}$ H. W. Choi, C. W. Jeon, M. D. Dawson, P. R. Edwards, and R. W. Martin, IEEE Photonics Technol. Lett. 15, 510 (2003).

${ }^{4}$ H. W. Choi, C. W. Jeon, M. D. Dawson, P. R. Edwards, and R. W. Martin, J. Appl. Phys. 93, 5978 (2003).

${ }^{5}$ Y. C. Shen, J. J. Wierer, M. R. Krames, M. J. Ludowise, M. S. Misra, F. Ahmed, A. Y. Kim, G. O. Mueller, J. C. Bhat, S. A. Stockman, and P. S. Martin, Appl. Phys. Lett. 82, 2221 (2003).

${ }^{6}$ H. W. Choi, M. D. Dawson, P. R. Edwards, and R. W. Martin, Appl. Phys. Lett. 83, 4483 (2003).

${ }^{7}$ S. H. Park, J. Jeon, Y. J. Sung, and G. Y. Yeom, Appl. Opt. 40, 3698 (2001)

${ }^{8}$ T. N. Oder, J. Shakya, J. Y. Lin, and H. X. Jiang, Appl. Phys. Lett. 82, 3692 (2003).

${ }^{9}$ J. F. Muth, J. D. Brown, M. A. L. Johnson, Z. Yu, R. M. Kolbas, J. W. Wood, Jr., and J. F. Schetzina, MRS Internet J. Nitride Semicond. Res. 4S1, G5.2 (1999). 